\title{
ANGIOGENIC RESPONSE INDUCED BY MECHANICAL TRANSMYYCARDIAL REVASCULARIZATION
}

Victor Chu, MD

Jin-qiang Kuang, MD

Amy McGinn ${ }^{\mathrm{a}}$

Adel Giaid, $\mathrm{PhD}^{\mathrm{b}}$

Stephen Korkola, MD

Ray C.-J. Chiu, MD, PhD ${ }^{\mathrm{a}}$
Background: Angiogenesis is the proposed mechanism of transmyocardial revascularization. We evaluated mechanical transmyocardial revascularization in a chronically ischemic porcine model by measuring myocardial angiogenic response. Methods: Ameroid constrictors were implanted 6 weeks before mechanical transmyocardial revascularization. Group I $(n=5)$ and group II $(n=3)$ animals received 30 punctures with an 18-gauge needle and samples were harvested at 1 and 4 weeks, respectively, after the operation. Group III $(n=5)$ had sternotomy only and served as the control group. Myocardial samples were immunohistochemically stained for vascular endothelial growth factor (VEGF), basic fibroblast growth factor (bFGF), and transforming growth factor $\beta$ (TGF- $\beta$ ) using specific antibodies. Growth factor expression was quantified by means of computer-assisted morphometry. Vascular density was assessed by immunohistochemical stain for VEGF and factor VIII. Results: Compared with group III, increased angiogenic factor levels were found in group I (VEGF $0.47 \pm 0.03 \mathrm{~mm}^{2}$ vs $0.05 \pm 0.05 \mathrm{~mm}^{2}, P$ $=.000 ;$ bFGF $0.67 \pm 0.14 \mathrm{~mm}^{2}$ vs $0.03 \pm 0.03 \mathrm{~mm}^{2}, P=.000 ;$ TGF $-\beta 1.40$ $\pm 0.18 \mathrm{~mm}^{2}$ vs $0.09 \pm 0.06 \mathrm{~mm}^{2}, P=0.000$ ), and in group II (VEGF 0.34 $\pm 0.06 \mathrm{~mm}^{2}$ vs $0.05 \pm 0.05 \mathrm{~mm}^{2}, P=.003$; bFGF $0.06 \pm 0.02 \mathrm{~mm}^{2}$ vs 0.03 $\pm 0.03 \mathrm{~mm}^{2}, P=.135$; TGF $-\beta 0.28 \pm 0.09 \mathrm{~mm}^{2}$ vs $0.09 \pm 0.06 \mathrm{~mm}^{2}, P=$ $.042)$. Vascular densities after mechanical transmyocardial revascularization were also increased (group I, VEGF stain $8.1 \pm 0.6$ vs $1.1 \pm 0.5, P$ $=.000$; factor VIII stain $5.1 \pm 2.7$ vs $0.4 \pm 0.3, P=.018$; group II, VEGF stain $1.9 \pm 0.5$ vs $1.1 \pm 0.5, P=0.107$; factor VIII stain $2.3 \pm 0.4$ vs $0.4 \pm$ $0.3, P=.004)$. Conclusions: Mechanical transmyocardial revascularization is associated with increased angiogenic factor expression and concomitant neovascularization at up to 4 weeks. These changes are indistinguishable from those of laser transmyocardial revascularization. Myocardial perfusion studies are needed to establish the functional significance of these angiogenic changes. (J Thorac Cardiovasc Surg 1999; 118:849-56)
$\Gamma \mathrm{r}$ ransmyocardial revascularization (TMR) is a newly developed surgical treatment for patients with symptomatic end-stage coronary artery diseases who have exhausted other treatment alternatives. Although

From the Division of Cardiothoracic Surgerya and the Department of Pathology, McGill University, ${ }^{\mathrm{b}}$ Montreal, Quebec, Canada.

Read at the Seventy-ninth Annual Meeting of The American Association for Thoracic Surgery, New Orleans, La, April 18-21, 1999.

Received for publication March 19, 1999; revisions requested May 4, 1999; revisions received July 8, 1999; accepted for publication July 13, 1999.

Address for reprints: Ray C.-J. Chiu, MD, Room C9.169, Montreal General Hospital, 1650 Cedar Ave, Montreal, Quebec, H3G 1A4, Canada (E-mail: mdiu@musica.mcgill.ca).

Copyright (C) 1999 by Mosby, Inc.

$0022-5223 / 99 \$ 8.00+0 \quad \mathbf{1 2 / 6 / 1 0 1 4 1 3}$ the mechanism of TMR is still controversial, a growing amount of clinical and experimental evidence suggests that TMR-induced angiogenesis plays an important role in this therapeutic approach.

Clinical development of TMR and its subsequent governmental approval has benefited immensely from close collaborations and financial support from the laser industry. In return, all clinical studies of TMR have used purposely designed laser devices since the early 1980s. However, the initially proposed advantage of laser, that is, improved long-term channel patency, is no longer supported by more recently available data. In fact, most investigators now believe that TMR achieves its therapeutic benefits independently of long-term channel patency.

Alternatively, it has been proposed that TMR induces 
angiogenesis and increases myocardial collateral circulation through a tissue injury/wound healing process. A logical extension of such a hypothesis is that angiogenesis is a nonspecific response to tissue injury, which can be created in a variety of ways. Indeed, in addition to a wide array of laser sources, radio frequency and simple needle punctures ${ }^{1}$ have also been shown to induce myocardial angiogenesis in animal models.

Mechanical needle puncture deserves further consideration, not only for its simplicity. It has also been shown by Sen and coworkers ${ }^{2}$ to be effective in the treatment of acute myocardial ischemia. More recently, Whittaker, Rakusan, and Kloner $^{3}$ demonstrated that needle punctures were more effective than laser channels in protecting acute ischemia in an animal model. Our earlier study ${ }^{4}$ also indicated that needle TMR compared favorably with a carbon dioxide laser. Nevertheless, the effectiveness of needle TMR in promoting myocardial angiogenesis and its potential as a simple and low-cost alternative to laser procedures have never been evaluated.

We performed needle mechanical TMR in a chronically ischemic porcine model. The purpose of this study is to further characterize the angiogenic response following needle TMR, providing a more detailed analysis with multiple growth factors over different time points.

\section{Methods}

All experimental animals were cared for in accordance with institutional guidelines and the "Guide for the Care and Use of Laboratory Animals" prepared by the Institute of Laboratory Animal Resources, National Research Council, and published by the National Academy Press, revised 1996.

Animal model with chronic ischemia. Fifteen Yorkshire pigs weighing 15 to $20 \mathrm{~kg}$ were premedicated with intramuscular ketamine $(15 \mathrm{mg} / \mathrm{kg})$ and were anesthetized with intravenous injection of thiopental sodium $(15 \mathrm{mg} / \mathrm{kg})$. After oral endotracheal intubation, anesthesia was maintained with $0.5 \%$ to $2.0 \%$ isoflurane in room air. Oxygen saturation was continuously monitored with a transcutaneous oximeter probe. Cefazolin, $500 \mathrm{mg}$, was given intravenously before the skin incision.

Animals were placed in the right lateral decubitus position. The thoracic area was prepared and draped in a sterile fashion. Exposure of the proximal left circumflex artery was achieved via a minithoracotomy through the 4th intercostal space. A 1-cm segment of the left circumflex artery before the first obtuse marginal branch was dissected free with both sharp and blunt dissections. Care was taken to minimize direct manipulation of the artery itself to avoid vessel spasm. An ameroid constrictor $(2.75 \mathrm{~mm}$, Research Instruments and Manufacturing, Corvallis, Ore) was placed around the left circumflex artery. The pericardium and the chest were closed in layers and the anesthesia reversed. The animals were kept for 6 weeks to allow time for gradual occlusion of the left circumflex artery by ameroid constrictors.

TMR. Six weeks after insertion of the ameroid constrictors, animals were randomly assigned to 3 groups ( $\mathrm{n}=5$ each). Groups I and II received 30 needle punctures whereas group III underwent sternotomy only. Tissue samples from groups I and III were harvested 1 week after the operation and samples from group II were harvested 4 weeks after TMR.

All TMR operations were performed through a median sternotomy. Anesthesia and intubation were performed in the same fashion as the first operation. All animals received a prophylactic intravenous lidocaine bolus $(2 \mathrm{mg} / \mathrm{kg}$ ) and were maintained on a lidocaine infusion $(1 \mathrm{mg} / \mathrm{min})$ throughout the operation. Median sternotomies were performed and the hearts were exposed by opening the pericardium and carefully dissecting away pericardial adhesions. Needle punctures were performed in an area measuring approximately $2 \times 2 \mathrm{~cm}$ between the 1st and 2nd obtuse marginal arteries with 18 gauge hypodermic needles. Transmural penetration was confirmed by noting pulsatile flow of arterial blood through the needle. Bleeding was controlled with finger pressure or 4-0 Prolene sutures (Ethicon, Inc, Somerville, NJ), which also served as markers of puncture sites at the time of tissue harvest. Sternums were then closed with steel wires and the incisions closed in layers. Anesthesia was reversed and the animal allowed to recover.

Sample harvest and cryopreservation. At the time of tissue harvesting, repeat sternotomies were performed through the same incision. Hearts were isolated by careful dissection of adhesions. Animals were put to death with an overdose of pentobarbital and potassium chloride. The ascending aortas were crossclamped and the hearts fixed in situ by injecting 1 $\mathrm{L}$ of ice cold $4 \%$ paraformaldehyde through the aortic root. Full-thickness slices of myocardium from the TMR-treated area (or corresponding ischemic area in the control group) were removed and immediately immersed in $4 \%$ paraformaldehyde in phosphate-buffered saline solution. These were kept at $4^{\circ} \mathrm{C}$ for 12 hours. The specimens were then transferred into $15 \%$ sucrose in phosphate-buffered saline solution and kept at $4^{\circ} \mathrm{C}$ for 3 days. Afterward, samples were embedded in OCT compound (Tissue-Tek, Sakura Finetek Inc, Torrance, Calif), snap frozen with liquid nitrogen, and kept at $-80^{\circ} \mathrm{C}$.

All ameroid constrictors were retrieved from the heart and inspected to confirm vessel occlusion.

\section{Sample analysis}

Immunohistochemistry. Cryostat sections of tissue samples were mounted on glass slides and immunostained with antisera to vascular endothelial growth factor (VEGF), basic fibroblast growth factor (bFGF), transforming growth factor $\beta$ (TGF- $\beta$ ) (Santa Cruz Biotechnology Inc, Santa Cruz, Calif), or factor VIII ligands (Sigma-Aldrich Canada Ltd, Oakville, Ontario, Canada) with a modified avidin biotin-peroxidase method. ${ }^{5}$ Tissue sections were made permeable with octylphenoxypolyethoxyethanol (Triton X-100; Union Carbide Corporation, Danbury, Conn), incubated in hydrogen 
peroxide to block endogenous peroxidase activity. They were then incubated first with normal goat serum for 30 minutes and followed with the primary antibody for 16 hours at $4{ }^{\circ} \mathrm{C}$. Afterward, they were incubated with biotinylated immunoglobulin $\mathrm{G}$ and stained with an immmunoperoxidase technique according to the manufacturer's instructions (Vectastain ABC Elite Kit; Vector Laboratories, Burlingame, Calif).

Angiogenic growth factor expression. Growth factor expression was quantified by measuring the area of tissue sections positively stained for VEGF, bFGF, or TGF- $\beta$ in each high-power field (400×). Measurements were performed around TMR puncture sites, which were identified by the following criteria: (1) identifiable needle or laser puncture scar under low-power view (100×), (2) presence of inflammatory cells and granulation tissue, and (3) loss of normal myocyte appearance and homogeneity. With the use of the sampling method of "systematic sampling with a random start," 8 sampling sites from each tissue section were photographed with a still video camera and digitized into tagged image file format (TIFF) files. Quantitative measurements of stained area were performed with an IBM compatible personal computer using Matrox Inspector 2.1 (Matrox Inc, Montreal, Quebec, Canada). Total amount of growth factor expression for each animal was reported as mean area of positive stain (square millimeters).

Vascular density. TMR-induced angiogenesis was quantified by measuring vascular density of VEGF- and factor VIII-stained blood vessels per high-power field around puncture sites. Positively stained vessels were defined as round structures with a central lumen, which was lined by a thin layer of endothelium stained positively for VEGF or factor VIII. Eight measurements were taken for each tissue section by means of the same sampling method. Results of angiogenesis for each animal were reported as mean number of vessels per high-power field.

Statistical analysis. All numeric data were reported as mean \pm 1 standard deviation where applicable. Data analyses with the Student $t$ test were performed with SPSS 7.5.2 for Windows software (SPSS Inc, Chicago, Ill).

\section{Results}

Mortality. Two deaths occurred in group II on postoperative day 2 after TMR. Autopsy showed severe pulmonary congestion and tissue edema with no evidence of myocardial infarction. The presumed cause of death was congestive heart failure.

Histology. All animals had complete occlusion of the left circumflex coronary artery at the time of sample harvesting. In only 1 of the 15 animals, a small area of transmural scar was noticed at the 2 nd operation before TMR. This was probably the result of early occlusion of the ameroid constrictor. In TMR-treated specimens, the areas of transmural punctures were easily identified by the presence of numerous fibrous scars on the endocardium.
Under low-power light microscopic examinations, transmural puncture sites could be identified as central fibrous tracts surrounded by inflammatory changes. All channels were completely occluded by fibrosis. One week after TMR (group I), the fibrous tracts consisted mainly of fibroblasts and collagen material with occasional small blood vessels. The surrounding area consisted of granulation tissue and damaged myocardium with infiltrating lymphocytes and macrophages. These were similar to the typical inflammatory changes during the normal tissue healing process. Numerous small vascular structures were also found in the area of tissue inflammation. These vessels were morphologically indistinguishable from native myocardial capillaries except for their endothelium, which was positively stained for VEGF or factor VIII (Fig 1). Most of these vessels were smaller than $10 \mu \mathrm{m}$ in diameter and were believed to be at various stages of angiogenic development.

Inflammatory changes related to needle punctures were limited to their immediate vicinity. Each puncture site was separated from others by normal-looking myocardium indistinguishable from the control specimen under light microscopy.

Four weeks after needle puncture (group II), the transmural channels were completely replaced by fibrous scar tissue. Significantly less inflammatory cellular infiltrate was observed in the granulation tissue surrounding needle tracts. Compared with group I, fewer vessels with positively stained endothelium were found in group II. However, the remaining vessels were more mature looking and had a somewhat larger diameter.

Angiogenic growth factor expression. Parallel comparison revealed that different angiogenic growth factors had distinct stain patterns (Fig 2). In samples taken 1 week after needle puncture (group I), several cell types stained positively for VEGF, including endothelial cells, macrophages, fibroblasts, and myocytes. In general, positive stains were limited to areas adjacent to the puncture sites. Endothelium and macrophages gave the most intense stains but represented only a small portion of the total area as measured by computer-assisted morphometry. On the other hand, myocytes and fibroblasts produced a more diffused staining pattern and represented most of our measurements for VEGF expression $\left(0.47 \pm 0.03 \mathrm{~mm}^{2}\right.$ vs $0.05 \pm 0.05 \mathrm{~mm}^{2} ; P<.001$; Fig 3, A). Staining was minimal in areas away from puncture sites, and measurements from these areas were not significantly higher than the baseline.

Four weeks after TMR (group II), there was less VEGF staining in the endothelial cells and macrophages. However, positive stain from myocytes and 

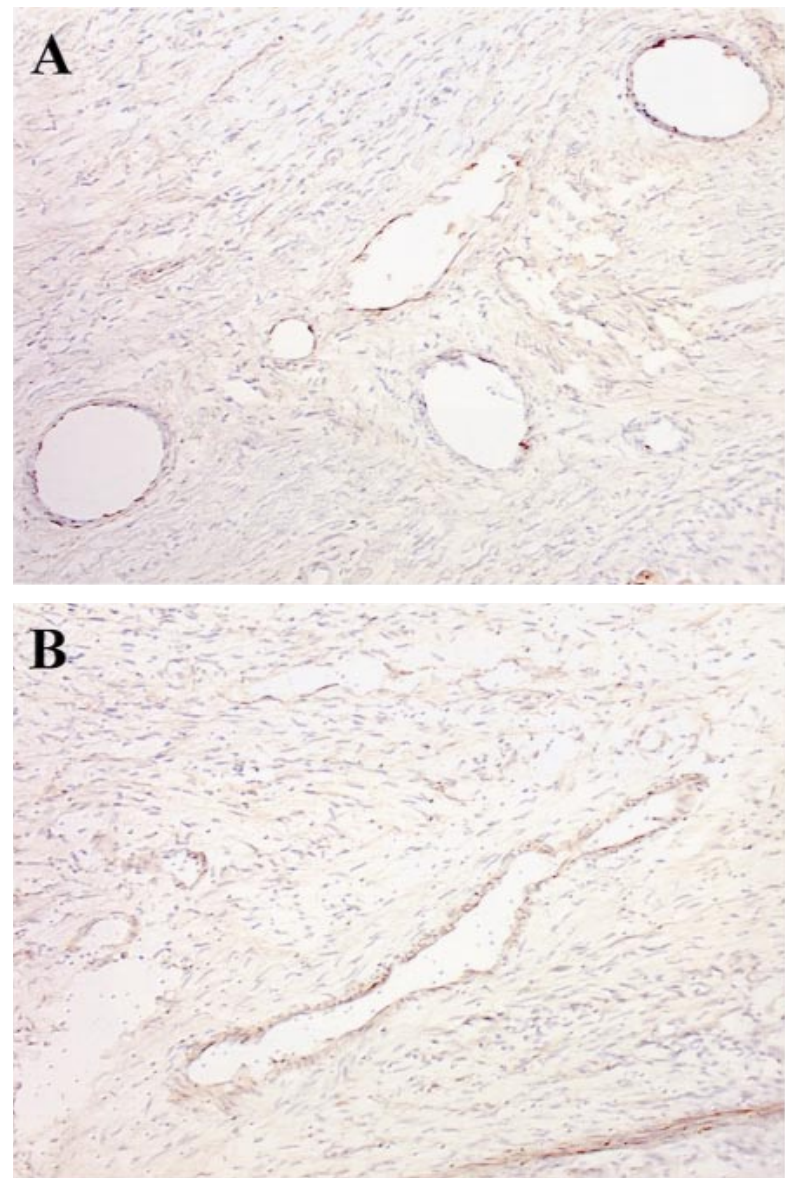

Fig 1. Representative images from TMR-treated area $(200 \times)$ illustrating increased number of small vessels with endothelium stained for VEGF (A) or factor VIII (B).

fibroblasts persisted, and the overall level as measured by morphometric analysis was still significantly higher than that of control specimens $\left(0.34 \pm 0.06 \mathrm{~mm}^{2}\right.$ vs $0.05 \pm 0.05 \mathrm{~mm}^{2} ; P=.003 ;$ Fig $3, A$ ).

Basic FGF stained much differently from VEGF. One week after TMR (group I), there was strong staining of fibroblasts along the needle tracts by anti-bFGF antisera $\left(0.67 \pm 0.14 \mathrm{~mm}^{2}\right.$ vs $0.03 \pm 0.03 \mathrm{~mm}^{2} ; P<0.001$; Fig $3, B)$. Very little stain was found in other cell types. The level of bFGF stain dropped to baseline at 4 weeks (group II, $0.06 \pm 0.02 \mathrm{~mm}^{2}$ vs $0.03 \pm 0.03 \mathrm{~mm}^{2} ; P=$ .135 ; Fig $3, B)$.

TGF- $\beta$ stain followed a pattern similar to that of VEGF 1 week after TMR (group I), that is, endothelial cells, macrophages, myocytes, and fibroblasts. Again, most of the morphometric measurement was from myocytes and fibroblasts, which gave a more diffuse stain pattern $\left(1.40 \pm 0.18 \mathrm{~mm}^{2}\right.$ vs $0.09 \pm 0.06 \mathrm{~mm}^{2} ; P$ $<.001$; Fig 3, C). At 4 weeks (group II), the level of
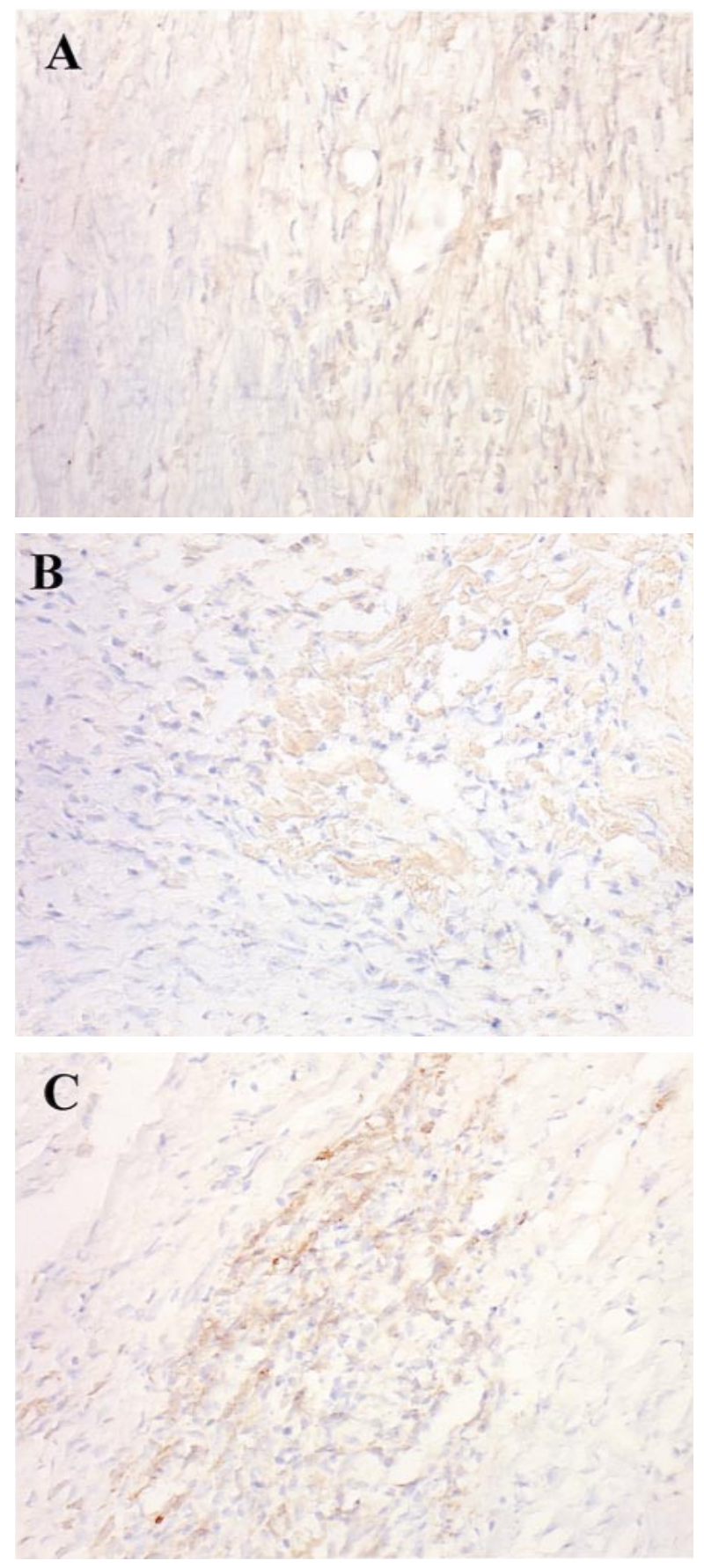

Fig 2. Representative digitized image of TMR-treated tissue section (200×) stained for VEGF (A), bFGF (B), and TGF- $\beta$ (C).

stain had decreased considerably but was still significantly higher than that of the baseline $\left(0.28 \pm 0.09 \mathrm{~mm}^{2}\right.$ vs $0.09 \pm 0.06 \mathrm{~mm}^{2} ; P=.042$; Fig $3, C$ ).

Angiogenesis. One week after needle puncture (group I), the number of vascular structures in the vicinity of needle punctures had increased significant- 

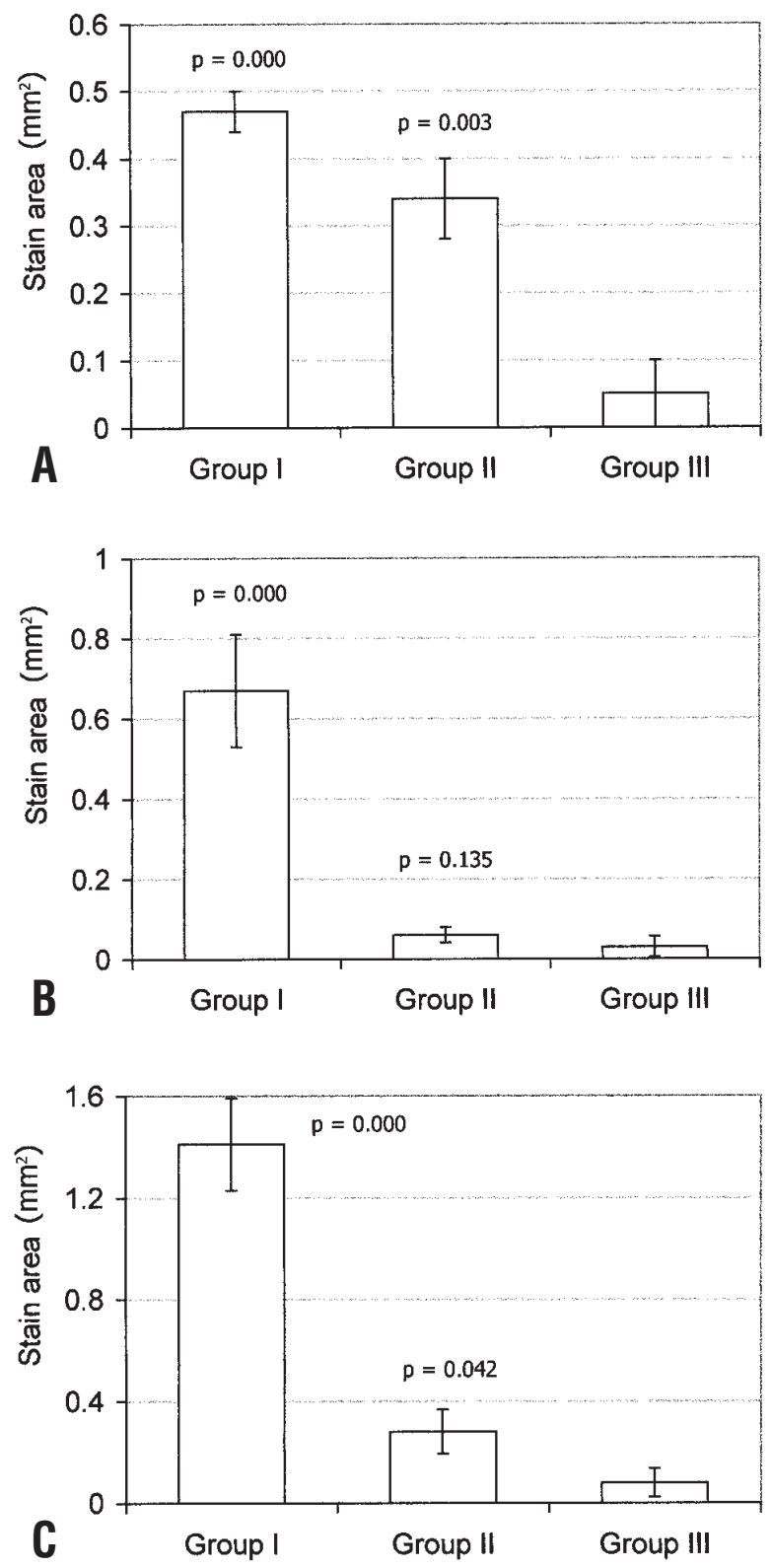

Fig 3. Comparison of angiogenic growth factor expression in all treatment groups. Growth factor expression is measured by mean area of VEGF stain $\left(\mathrm{mm}^{2}\right)$ per high-power field. Error bars represent 1 standard deviation. $P$ values are comparisons against group III. A, VEGF; B, bFGF; $\mathbf{C}$, TGF- $\beta$.

ly. This increase in vascular density was found by both VEGF staining $(8.1 \pm 0.6$ vs $1.1 \pm 0.5 ; P<.001)$ and factor VIII staining $(5.1 \pm 2.7$ vs $0.4 \pm 0.3 ; P=.018)$. These vessels were mostly between 2 and $10 \mu \mathrm{m}$ in diameter and were believed to be at various stages of angiogenic development (Fig 1).

The number of VEGF-stained vessels dropped to baseline at 4 weeks $(1.9 \pm 0.5$ vs $1.1 \pm 0.5 ; P=.107)$,
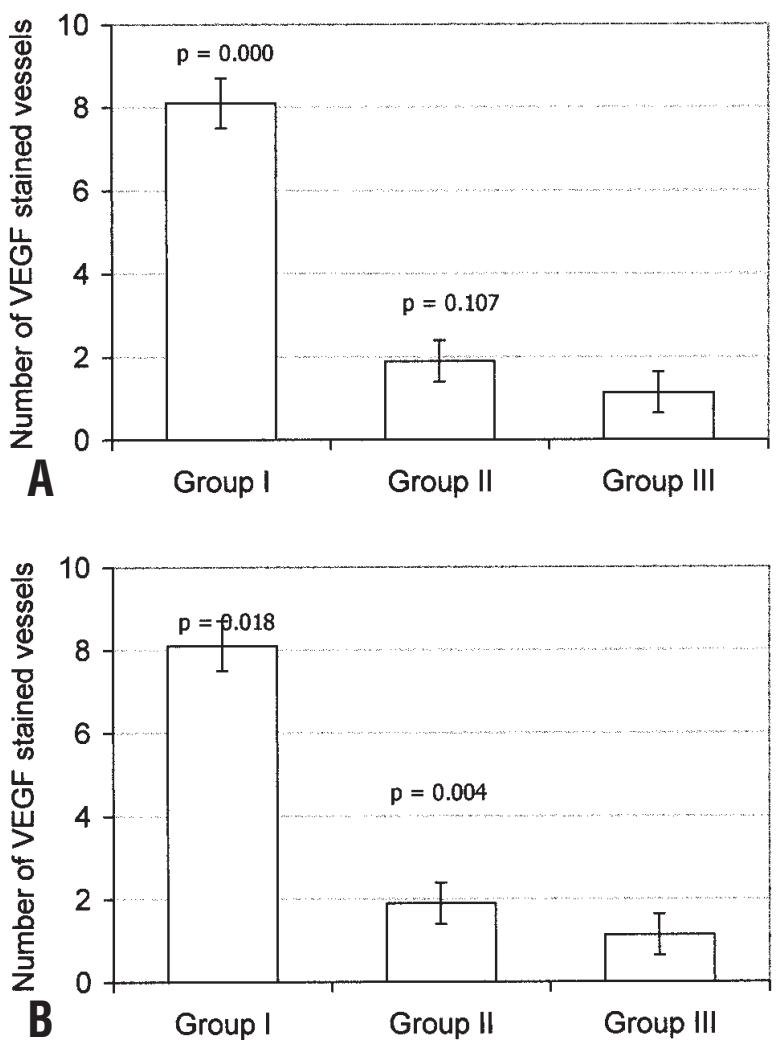

Fig 4. Comparison of vascular densities. Mean number of vessels per high-power field is shown in the Y-axis. Error bars represent 1 standard deviation. $P$ values are comparisons against group III. A, VEGF; B, factor VIII.

and the number of factor VIII-stained vessels was lower but still significantly higher than that of the control specimens $(2.3 \pm 0.4$ vs $0.4 \pm 0.3 ; P=.004)$. Comparison of vascular densities is shown in Fig 4.

\section{Discussion}

Since it was first introduced by Mirhoseini and Cayton, ${ }^{7}$ all clinical studies of TMR have used laser devices. This is not surprising, since the laser industry has been instrumental in providing technical and financial support for this procedure from the beginning. Although no evidence has been produced to substantiate the proposed benefit of laser, laser TMR remains the only procedure accepted in clinical practice. Without a clear understanding of its mechanism, this situation will likely remain unchanged despite the technical complexity and significant cost associated with laser TMR.

Debates over the basic mechanism of TMR have a history almost as long as that of the procedure itself. The initial "open-channel" hypothesis based on a reptilian-style transmural perfusion is no longer supported 
by the currently available research data. Not only is there a lack of evidence indicating the existence of patent channels beyond the immediate postoperative period, but the potential amount of blood flow through these channels is also insignificant because of physiologic limitations. ${ }^{89}$ More recently, several other hypotheses have been proposed in an effort to explain the apparent effectiveness of TMR in reducing angina symptoms, among these, angiogenesis.

TMR-induced angiogenesis is based on the observation that significant inflammatory reaction is consistently present in the vicinity of myocardial punctures. ${ }^{10-13}$ Angiogenesis and neovascularization play a central role during the initial phases of wound healing. ${ }^{14}$ This angiogenic response is stimulated by various growth factors released as a result of tissue injury and inflammatory cellular infiltration. The end result is seen as increased vascular density in the injured area. Central to this angiogenic hypothesis is that the release of angiogenic growth factors is a nonspecific response to tissue injury, which can be created by a variety of methods. Although laser was initially used to improve long-term channel patency, perhaps its true value is simply being a glamorous way to incite myocardial injury. In fact, a recent study of TMR by radio frequency ablation in a porcine model of chronic ischemia also showed increased angiogenesis. Simple needle punctures of myocardium have also been shown to induce an angiogenic response in an acute ischemic rat model. ${ }^{1}$ More recently, we demonstrated that 1 week after TMR, treatments with both needle and carbon dioxide laser punctures resulted in a similar increase in the expression of VEGF and tissue vascular density. ${ }^{4}$ Armed with these encouraging preliminary results, we took a closer look at mechanical needle TMR as a potential alternative to laser TMR.

Several observations from our study are consistent with the hypothesis of injury-induced angiogenic response. One week after a mechanical TMR procedure, an intense inflammatory reaction surrounded the myocardial puncture sites, which is characteristic of a wound healing process. The intensity of tissue inflammation was markedly reduced by 4 weeks after the operation as the healing process has moved from the inflammatory toward the proliferating phase. As expected, different growth factors had distinct stain patterns, which is a reflection of their intrinsic protein syntheses and ligand bindings. All 3 growth factors had a significantly elevated level of expression at 1 week. At 4 weeks, the levels of VEGF and TGF- $\beta$ decreased significantly as a result of diminished inflammatory cellular infiltrates and less staining of myocytes and fibro- blasts. However, their levels remained elevated when compared with those of control specimens. On the other hand, bFGF stain was mainly limited to the fibroblasts along needle puncture sites, and its level returned to baseline with maturing of the scar tissue. Increased angiogenic growth factor expression was limited to the region of myocardial punctures. In samples taken from the interventricular septum of group I and group II animals, the levels of angiogenic factors and vascular densities were similar to those of group III animals.

VEGF is a potent direct stimulant of neovascularization and vessel proliferation with receptors on the endothelial cells. Significantly higher number of developing vessels with VEGF-stained endothelium were found at 1 week. We postulate that these were newly formed vessels stimulated by locally secreted VEGF. When sections from the 4-week group were analyzed, the number of VEGF-stained vessels had decreased to that of the baseline. However, this was mostly due to decreased VEGF binding to its endothelial receptors rather than disappearance of newly formed vessels. Staining with factor VIII confirmed that vascular density was reduced but still significantly higher than that of control samples. Although the level of angiogenic growth factors will gradually return to baseline after mechanical TMR, some of the newly formed vessels will likely remain open in the presence of tissue ischemia.

TMR angiogenesis occurs at the expense of myocardial injury. It is important to take into consideration the issue of TMR "efficiency," which could be defined as the level of angiogenesis at a given amount of muscle destruction. Whether mechanical trauma by needle punctures or laser thermal injury is more efficient in creating angiogenesis remains to be clarified by future studies.

In summary, our study demonstrated that mechanical needle TMR is effective in stimulating intrinsic expression of several different angiogenic growth factors, and these findings were fundamentally indistinguishable from other studies of TMR using laser devices. In view of this and the significant cost implication, we believe that it is justifiable and perhaps desirable to include a mechanical needle TMR arm for comparison with laser TMR in future clinical trials.

Two questions remain to be answered by this study: What is the fate of mechanical TMR-induced vessels beyond 1 month? What is the functional significance of these vessels? Future studies with longer follow-up time, as well as functional and perfusion studies of mechanical TMR-treated myocardium, would help to answer these questions. 
We thank Dianne Murray and Marlene Brydon for their dedication in animal care and Jean-Yves Latreille, RN, for technical assistance in operating laser equipment.

\section{REFERENCES}

1. Pelletier MP, Giaid A, Sivaraman S, et al. Angiogenesis and growth factor expression in a model of transmyocardial revascularization. Ann Thorac Surg 1998;66:12-8.

2. Sen PK, Daulatram J, Kinare SG, et al. Further studies in multiple transmyocardial acupuncture as a method of myocardial revascularization. Surgery 1968;64:861-70.

3. Whittaker P, Rakusan K, Kloner RA. Transmural channels can protect ischemic tissue: assessment of long-term myocardial response to laser- and needle-made channels. Circulation 1996; 93:143-52.

4. Chu VF, Giaid A, Kuang J-Q, et al. Angiogenic response in transmyocardial revascularization: comparison of laser vs. mechanical punctures. Ann Thorac Surg. In press.

5. Hsu S, Raine L, Fanger H. Use of avidin-biotin-peroxidase complex $(\mathrm{ABC})$ in immunoperoxidase techniques: a comparison between $\mathrm{ABC}$ and unlabeled antibody (PA) procedures. J Histochem Cytochem 1981;29:577-80.

6. Weibel E. Stereological methods. 1st ed. Vol 1. New York: Academic Press; 1979.

7. Mirhoseini M, Cayton MM. Revascularization of the heart by laser. J Microsurg 1981;2:253-60.

8. Hardy RI, James FW, Millard RW, Kaplan S. Regional myocardial blood flow and cardiac mechanics in dog hearts with $\mathrm{CO}_{2}$ laser-induced intramyocardial revascularization. Basic Res Cardiol 1990;85:179-97.

9. Kohmoto T, Fisher PE, Gu A, et al. Does blood flow through holmium:YAG transmyocardial laser channels? Ann Thorac Surg 1996;61:861-8.

10. Jansen ED, Frenz M, Kadipasaoglu KA, et al. Laser-tissue interaction during transmyocardial laser revascularization. Ann Thorac Surg 1997;63:640-7.

11. Whittaker P. Detection and assessment of laser-mediated injury in transmyocardial revascularization. J Clin Laser Med Surg 1997;15:261-7.

12. Krabatsch T, Schaper F, Leder C, et al. Histological findings after transmyocardial laser revascularization. J Card Surg 1996;11: 326-31.

13. Fleischer KJ, Goldschmidt-Clermont PJ, Fonger JD, et al. Onemonth histologic response of transmyocardial laser channels with molecular intervention. Ann Thorac Surg 1996;62:1051-8.

14. Davidson J. Wound repair. In Gallin J, Goldstein I, Snyderman R, editors. Inflammation: basic principles and clinical correlates. New York: Raven Press; 1992. p. 809-19.

\section{Discussion}

Dr Ernst Wolner (Vienna, Austria). In 1969 I published an experimental study very similar to yours, with ameroid constrictors, but I did not use this puncture. That was the time of the Vineberg operation. I used strips from the jugular vein and a curved needle, and I positioned 7 to 8 of these strips on the left anterior wall, from the outside to the inside of the cavity and back to the outside. At that time we did not have growth factor, but we did find some new vessels.

Using xenon 133, we also made some overall measure- ments of blood flow on the anterior wall of the left ventricle. We found an increase in the overall blood supply or blood perfusion of the anterior wall. Thus these old experiments are in complete accordance with your data, indicating that it seems to be enough to create an injury to get some changes and not to use this very expensive laser.

Dr Chu. Thank you, Dr Wolner.

Dr Thomas M. Egan (Chapel Hill, NC). That was a very nice presentation. I wonder about the accuracy of using immunohistochemical staining for your outcome measures. Specifically, how did you deal with background expression? Have you made an attempt to measure messenger RNA instead of relying on expression?

Dr Chu. These are very good points. First of all, to examine the presence of background angiographic expression, we stained tissue sections not only from sham-operated animals, which is our control group, but also from unpunctured areas in our treatment groups. Although these results were not shown here, we found that the levels of immunostaining from unpunctured areas were consistently low and similar to those of our control group. It seems that background expression is not a big problem.

Messenger RNA level was not measured in this particular study.

Dr Beat H. Walpoth (Bern, Switzerland). It seems that you did not do TMR and needle puncture in the same animal but rather did a historical comparison. You did not present results comparing the 2 techniques. You just showed that needle puncture will induce growth factors. I think this point is worth mentioning, although it has been noted before.

An interesting question, however, is to know whether laserinduced injury, yielding a stronger and bigger injury, will induce quantitatively more growth factors. Can you comment?

Dr Chu. Absolutely. In fact, this study was an extension of our preliminary experiments, which were presented before the Society for Thoracic Surgeons meeting in San Antonio. In that study, we directly compared the effect of needle punctures versus carbon dioxide laser TMR. I agree that individual laser puncture causes much more tissue damage and therefore more angiogenic factor expression per puncture. However, we also showed that such difference was only quantitative and could be fully compensated by simply increasing the number of needle punctures.

There is a balance between tissue damage and angiogenic response, and I cannot tell you whether needle or laser is more efficient. However, the bottom line is that the same level of angiogenesis can be achieved using simple needle punctures.

Dr Jakob Vinten-Johansen (Atlanta, Ga). Is there a cause-and-effect relationship between the local expression of angiogenic factors and the actual capillary density that is observed?

Dr Chu. We definitely found that tissue capillary densities parallel the levels of various angiogenic factors. In addition, we found an increased number of vessels in needle-treated areas with VEGF-stained endothelium. We think these could be locally produced protein products binding to endothelial 
receptors. Yes, we believe there is a cause-and-effect relationship.

Dr Paul Kurlansky (Miami Beach, Fla). Dr Chu, I am following your work with interest. I saw your presentation at the Society for Thoracic Surgeons meeting. My question regarding needle puncture versus laser TMR is as follows: Have you done any studies on the time course and the quality of the effect in addition to the work you have shown us on the quantity of the effect? In other words, is there a similar time course as regards neovascularization and VEGF expression, and are there other growth factors that are expressed differentially with one technique versus the other?
Dr Chu. Thank you very much. This study was meant to further characterize angiogenesis of mechanical needle TMR only. We included 2 animal groups at 1 and 4 weeks postoperatively for the purpose of time-course study. We also looked at several different angiogenic factors, as well as including factor VIII for measuring vascular density. Indeed, the patterns of expression and the time course of different angiogenic factors were quite distinct from one another.

I think an important question here is whether the increased vascular density has any functional significance. This will be answered by myocardial perfusion studies currently in progress in our laboratory. 